\title{
Re: Cytoreductive Radical Prostatectomy in Patients with Prostate Cancer and Low Volume Skeletal Metastases: Results of a Feasibility and Case-Control Study
}

\author{
Axel Heidenreich, David Pfister, Daniel Porres
}

Uniklinik RWTH Aachen, Department of Urology, Aachen, Germany

J Urol 2015;193:832-838.

\section{EDITORIAL COMMENT}

Androgen deprivation therapy (ADT) is currently the standard treatment for patients with metastatic prostate cancer (mPCa) (1). Survival improvement after cytoreductive surgery is well known for kidney, breast, colon and ovarian cancers. The survival benefit of cytoreductive radical prostatectomy (CRP) for mPCa was first hypothesized in a SEER-based study (2). Heidenreich et al. recently reported survival outcomes of CRP for mPCa. In the present study, $23 \mathrm{mPCa}$ patients with less than 3 bone metastases, no visceral or extensive LN metastases and PSA less than $1.0 \mathrm{ng} / \mathrm{mL}$ after ADT were included. Control group (CG) consisted of $38 \mathrm{mPCa}$ patients treated with ADT alone. CRP and CG were equal with respect to patient demographics, tumor characteristics, and comorbidities. All patients had $50 \mathrm{mg}$ bicalutamide with LHRH agonists at least for 6 months before surgery. Patients, who did not have PSA progression, were recommended to continue another 2 years of ADT before treatment stopped. When compared with CG, median time to castration resistance was statistically significantly longer in CRP group (40 vs 29 months, $p=.014)$. At a median follow-up of 3 years, overall survival rates were $91.3 \%$ and $78.9 \%$ for $C R P$ and $C G$, respectively ( $p=0.048$ ). Cancer-specific survival rates were $95.6 \%$ and $84.2 \%$ for $C R P$ and $C G$, respectively ( $p=0.043$ ). Median clinical progression-free survival (PFS) (range) was 38.6 and 26.5 months in $C R P$ and $C G s$, respectively ( $p=0.032$ ). This was the first case-control study that showed that CRP combined with ADT might offer better survival outcomes than ADT alone in mPCa patients. In spite of retrospective design and small cohort, the results are promising. There is an ongoing clinical trial testing the treatment efficacy of primary tumor in mPCa patients. This trial is going to compare efficacy of ADT therapy and ADT combined with either CRP or radiation (NCT01751438). SEER study was not a case-control study. This study further enhanced the hypothesis of the SEER study, and thus, these results may increase urologists' role in multi-modal treatment approaches.

\section{REFERENCES}

1. Heidenreich A, Bastian PJ, Bellmunt J, Bolla M, Joniau S, van der Kwast T, Mason M, Matveev V, Wiegel T, Zattoni F, Mottet N. EAU guidelines on prostate cancer. Part II: Treatment of advanced, relapsing, and castration-resistant prostate cancer. Eur Urol 2014;65:467-479.

2. Culp SH, Schellhammer PF, Williams MB. Might men diagnosed with metastatic prostate cancer benefit from definitive treatment of the primary tumor? A SEER-based study. Eur Urol 2014;65:1058-1066.

Tayyar Alp Özkan, MD 